\title{
ACTIVE GALACTIC NUCLEI: VARIABILITY AT MANY WAVELENGTHS
}

\author{
THIERRY J.-L. COURVOISIER \\ INTEGRAL Science Data Centre \\ 16, ch. d'Ecogia \\ CH-1290 Versoix \\ Switzerland \\ and \\ Observatoire de Genève \\ CH-1290 SAUVERNY \\ Switzerland
}

\section{Introduction}

Active Galactic Nuclei (AGN) are thought to be powered by accretion onto a massive black hole. Understanding how gravitational energy freed by the infall of matter into the black hole is transferred to the radiating regions of the nucleus is one of the main challenges of AGN research. The question is made very complex by the presence of several cooling mechanisms with very diverse physical properties: We observe synchrotron radiation, thermal emission from hot dust, possibly thermal optical and ultraviolet emission and Comptonization processes in the $\mathrm{X}$-ray domain. For each component the radiation is a signature of the cooling process rather than of the heating process. It is our hope that by observing the links and correlations between the emission of the different components we will be able to understand how they are interrelated and how they get their energy supply from the accretion process.

We briefly review here how the UV and X-ray variability depends on the luminosity of the objects and give a progress report on the cross correlations that we can deduce from more than 10 years of extensive observations of the quasar $3 \mathrm{C} 273$.

\section{Ultraviolet and X-ray Variability of samples of AGN}

(Paltani and Courvoisier 1994) analysed the variability of all the AGN observed several times by IUE. We found that the average variation of the sources in each of the AGN classes (radio loud quasars, radio quiet quasars, Seyfert 1 galaxies, BL Lac objects; approximately 20 objects per class) are very similar. I.e., the average amplitude of the variations of the sample of sources in each subclass is the same. More surprisingly, the wavelength dependence of the variations is also not a function of the class of objects. This result is not expected for sources which are thought to be powered in the ultraviolet domain by processes as different as synchrotron radiation in a jet (BL Lac objects) or by a heated accretion disk (Seyfert 1 galaxies).

These data were also used to study the variability at $1200 \AA$ in the rest frame of the objects for all non BL Lac objects (Paltani and Courvoisier 1997). It is found that the amplitude of the variations depends on the luminosity of the object $L$ like $L^{-0.08}$. This result cannot be interpreted in the frame of models in which the luminosity of the AGN is due to the incoherent addition of events, unless the average properties of the event in any one AGN depend on its average luminosity. (Courvoisier et al. 1996) have suggested a model based on stellar collisions in the vicinity of a supermassive black hole which qualitatively satisfies this condition.

A recent analysis of the objects observed several times in the X-rays by EXOSAT and in which the variability is defined in a manner similar to that used in the ultraviolet domain gave the same 


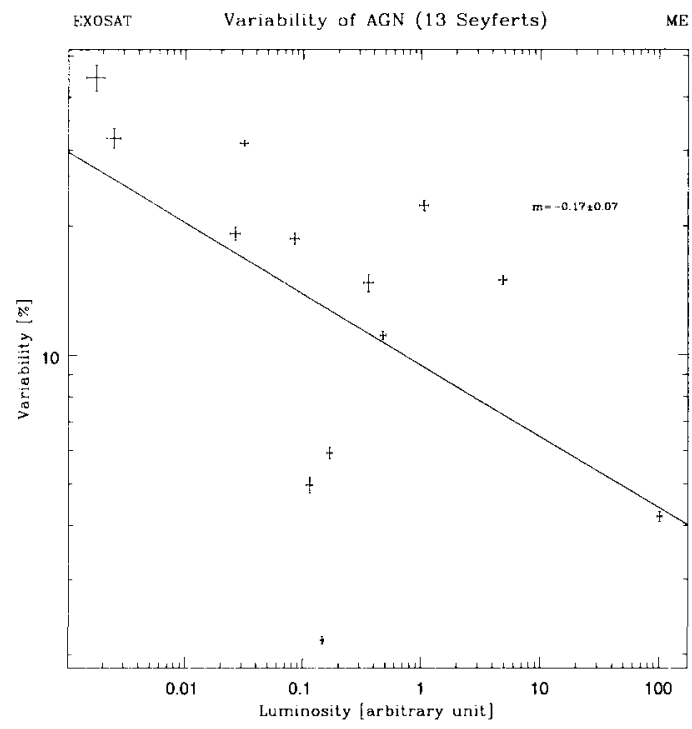

Figure 1. The amplitude of the X-ray variability of a set of sources observed several times with EXOSAT as a function of the luminosity. The observed correlation (straight line) indicates that the amplitude of the variations depends on the luminosity of the object.

result (see figure) for the luminosity dependence of the variability. As in the UV domain the slope of the dependence is not compatible with the incoherent addition of events.

\section{Multi-wavelength cross correlations in $3 \mathrm{C} 273$}

We have gathered in Geneva data obtained with several ground based and space born instruments. Figure 2 gives an overview of the data available as a grey scale image, the axes are the frequency and the epoch of the observation. The grey intensity is a measure of $\nu \cdot F_{\nu}$.

Using these data we can deduce several results on cross correlations. This work is in progress and will be submitted for publication in late 1997. I present here some preliminary conclusions.

\subsection{OPTICAL-UV BANDS}

There is a very good correlation at very short lags (typically less than 10 days) between the light curves obtained with both IUE cameras and the optical photometry. This result confirms the analysis given by (Courvoisier and Clavel 1991). Work is in progress to analyse whether the small lags are significantely different from 0 . The shortness of the lags between the emission in the UV and optical bands, i.e. between regions of different temperatures, has been one of the main motivations for models in which the accretion disc is heated by an external source rather than by the local release of gravitational energy (Courvoisier and Clavel 1991).

Analysis of the structure function of the light curves also reveals that the variability at long optical wavelengths extends to longer timescales than that observed in the ultraviolet domain ((Paltani 1995) and work in progress).

\subsection{UV AND RADIO BANDS}

We have previously noted (Courvoisier et al. 1990) that the ultraviolet light curve is well correlated with the radio light curves. We noted that although this had not been expected it is a natural correlation if we consider that the UV emission is a signature of the accretion process. In this case 


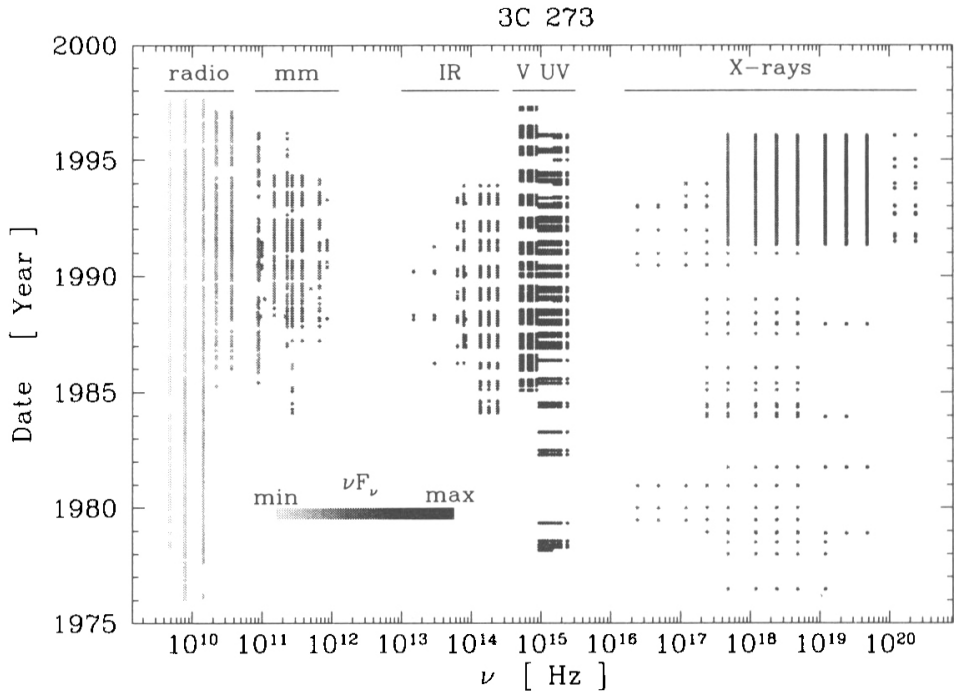

Figure 2. $\quad \nu \cdot f_{\nu}$ image for the bright radio loud quasar 3C 273 from $10^{10} \mathrm{~Hz}$ to $10^{19} \mathrm{~Hz}$ and from 1978 to 1997 (the complete life of IUE)

the radio synchrotron emission will result from energy carried to the relativistic electrons probably along the jet. This energy transport takes some time: the lag we observe between the two light curves. This result is still present in the longer set of data now available. It appears, however, that not all the features observed in the UV light curves are matched with corresponding structures in the radio light curves. This shows that, provided that the UV emission is indeed a signature for the accretion process, the energy is channeled to the radio domain in a way that is not always the same. I.e., the energy is distributed in the various cooling channels in a different way in different accretion events.

Taking the observed time delay (0.4y; (Courvoisier et al. 1990) and work in progress) and assuming that the energy is indeed carried along the jet at the velocity of the VLBI jet and in the direction of the jet $(\beta=\cos \theta=0.95$; (Davis et al. 1991)). We obtain that the radio emission at 22 $\mathrm{GHz}$ is located at 4 l.y. from the accretion site.

\subsection{X-RAY AND UV LIGHT CURVES}

The X-ray data set can now include the pointed observations obtained by a series of satellites in the past decades and publically available BATSE light curves. The resulting sampling is very inhomogeneous and the data from the different instruments must be extrapolated to a single energy. This extrapolation can be done with some confidence in $3 \mathrm{C} 273$, because the hard X-ray component is a single power law with an index that shows small (but significant) variations. The resulting light curve may then be cross-correlated with the UV light curves. The full X-ray data set, but also only the Batse data, are sufficient to show that the X-ray emission is only very poorly correlated with the UV light curve at 0 lag. This is in contrast to results obtained in multi-wavelength campaigns of observations of Seyfert galaxies (e.g. NGC 4151; (Edelson et al. 1996)), where it is found that the X-ray and UV light curves are well correlated with short lags.

The result we obtain in the case of $3 \mathrm{C} 273$ indicates that if the UV is due to reprocessed X-rays, it must be in such a way that the heating light curve does not match the X-rays observed far away.

A mathematically significant correlation is found between the two light curves, the $\mathrm{X}$-rays following the UV emission by about 1.2 years. This effect is, however, produced by one large event. 
The lag is therefore physically meaningful only if the UV and the X-ray events are indeed related.

\subsection{X-RAY AND RADIO LIGHT CURVES}

Cross-correlations of radio $(22 \mathrm{GHz}$ and $37 \mathrm{GHz})$ and $\mathrm{X}$-ray light curves clearly indicate that at 0 lag the two light curves are not correlated. This result confirms that described in (Courvoisier et al. 1990). The two light curves are, however correlated for a lag such that the X-rays follow the radio emission by approximately 2.2 years. As in the previous subsection, this effect is based on one large X-ray event, the same caveat, therefore applies.

The lack of correlation at short lags excludes that the X-rays are a Comptonised synchrotron component in which the Comptonisation would be due to the same electrons that produce the synchrotron emission. This would indeed lead to a tight correlation between both fluxes at zero or very short lags.

\section{Conclusions}

Models in which the UV emission is based on any event description must either include a mechanism through which the average properties of the events depend on the average luminosity of the object or mechanisms that contrive the events to be interdependent in a very specific way. The former may be achieved if the event properties depend on the mass of the central black hole. The same is probably true for the $\mathrm{X}$-ray domain, although a firm result will have to wait until light curves from a fair sample of objects can be obtained. It is interesting to note that (Leighly 1997) have also come to the conclusion that at least some X-ray light curves cannot be produced by the linear superposition of events.

There is no one to one correspondence between "events" observed in the different wavebands in the long term light curves of 3C 273 . This means that the energy is distributed in different ways among the radiation channels of the AGN at different epochs. The mechanism which operates this distribution is not known. This is probably the reason for which it is difficult to extract a consistent model of the sources from multi-wavelength data sets.

Taken at face value the multi-wavelength data available on $3 \mathrm{C} 273$ indicates that the UV emission could lead all the other components. This result which is in strong need of confirmation (through a long and properly sampled sets of light curves covering the radio to hard X-ray bands) is expected if the UV is a signature of the accretion process and hence of the energy available. In this case, the lags will prove a powerful tool to entangle the geometry of the source.

\section{Acknowledgemets}

It is a pleasure to acknowledge discussions on the issues presented here with $\mathrm{R}$. Walter, S. Paltani, M. Türler, M. Polletta and M. Audard.

\section{References}

Courvoisier T.J.-L. et al., 1990, A\&A 234, 73

Courvoisier T.J.-L. and Clavel J., 1991, A\&A 248, 389.

Courvoisier T.J.-L., Paltani S. and Walter R., 1996, A\&A 308 L17

Davis R.J., Unwin S.C. and Muxlow T.W.B., 1991, Nature 354, 374

Edelson R.A., et al., 1996, Ap.J. 470, 364

Leighly K., 1997, these proceedings

Paltani S., 1995, Ph.D. thesis, Geneva University

Paltani S. and Courvoisier T.J.-L., 1994, A\&A 291, 74

Paltani S. and Courvoisier T.J.-L., 1997, A\&A 323, 717 